\title{
Multicrystal harmonic generator compensates for thermally induced phase mismatch
}

Sheng Wu, Geoffrey A. Blake, Sunny Sun, Henry Yu, John Ling

Sheng Wu, Geoffrey A. Blake, Sunny Sun, Henry Yu, John Ling, "Multicrystal harmonic generator compensates for thermally induced phase mismatch," Proc. SPIE 3928, Nonlinear Materials, Devices, and Applications, (23 March 2000); doi: 10.1117/12.379920

SDIE Event: Symposium on High-Power Lasers and Applications, 2000, San Jose, CA, United States 


\title{
Multicrystal harmonic generator compensates for thermally induced phase mismatch
}

\author{
Sheng Wu', Geoffrey A. Blake ${ }^{1}$ \\ Sunny Sun, Henry Yu, John Ling ${ }^{2}$ \\ ${ }^{1}$ Division of Geological and Planetary Sciences, California Institute of Technology, CA 91125 \\ ${ }^{2}$ CASIX Inc., 21828 \#D, Lassen St. Chatsworth, CA 91131
}

\begin{abstract}
We use computer simulation to illustrate how thermally induced phase mismatch affect deep UV harmonic generation. A multicrystal harmonic generator that compensates for thermally induced phase mismatch is then presented. We have tested this multicrystal design with a Nd:YAG lasers $(1064 \mathrm{~nm}) 4$ th harmonic generator based on two pieces of $\beta-\mathrm{BaB}_{2} \mathrm{O}_{4}(\mathrm{BBO})$ crystals, and our results demonstrate that it compensates for the thermally induced phase mismatch, effectively increasing the interaction length of nonlinear optical crystals during harmonic generation under high loading.
\end{abstract}

Key words: multicrystal harmonic generator, $\mathrm{BBO}$, thermally induced phase mismatch

\section{INTRODUCTION}

There is a great demand for all solid state, high power UV lasers because of their compactness, wide tunability, ease of operation and efficiency. However, the average power output of such all solid state UV lasers is still well below that available from excimer lasers. One problem that limits the UV output of all solid state lasers is the lack of efficient and durable nonlinear optical (NLO) crystals that can generate harmonics deep in the UV. This problem is being solved with the discovery of materials such as $\beta-\mathrm{BaB}_{2} \mathrm{O}_{4}(\mathrm{BBO})$ and $\mathrm{CsLiB}_{6} \mathrm{O}_{10}(\mathrm{CLBO})$. The second concern is the increasing UV absorption in these crystals with decreasing wavelengths, which generates thermally induced phase mismatch and limits the interaction length of the nonlinear optical crystals ${ }^{1,2.3 .4 .5}$.

To solve the problem of thermally induced phase mismatch, several approaches have been proposed and tested, such as laser beam fanning/scanning ${ }^{6}$ or an N-Plate crystal configuration ${ }^{7}$ to promote heat dissipation. These earlier experiments were designed for crystals which have strong absorption at the input fundamental wavelength (1064 $\mathrm{nm}$ in this case), and therefore uniform heat dissipation along the laser propagation direction. Laser beam cylindrical focusing and collimating schemes ${ }^{8.9}$ have also been used to distribute the heat generated over a larger area. More recently, an external cooling method has also been developed which uses external gas cooling to compensate for the phase mismatch induced by the high temperature at the end of the crystal ${ }^{1}$.

These recent methods all attempt to remove heat generated as a result of the UV harmonics, which are absorbed much more strongly than are the input fundamentals (e.g. $532 \mathrm{~nm}$ and $1064 \mathrm{~nm}$ ) in materials like BBO and CLBO. Here, we propose a multicrystal design which passively compensates for the thermally induced phase mismatch, and thereby generates higher efficiency and better repeatability.

The thermally induced phase mismatch is a result of the temperature gradient formed inside the crystal. As demonstrated in the adjacent manuscript in this proceeding, NLO crystals have much stronger two photon absorption (TPA) in the presence of intense deep UV photons. Therefore, the heat generated inside the single crystal is not uniform, and the temperature gradient will be close to a 2 nd order parabolic with its maximum close to the output face of the crystal if no external cooling is applied. As shown by two plots below and other plots in the adjacent manuscript, it is noticed that besides the temperature gradient, there is also an average temperature rise inside the bulk crystal. The average temperature rise is much larger than the

Correspondence: sheng@its.caltech.edu

In Nonlinear Materials, Devices, and Applications, Jeffrey W. Pierce, Editor

Proceedings of SPIE Vol. $3928(2000) \bullet 0277-786 \times 100 / \$ 15.00$ 
temperature gradient, but it could be compensated by adjusting the phase matching angle in a certain direction. But when the laser is operating and if the thermal load is very high, then the average temperature rise will be much larger than the temperature bandwidth of the particular NLO crystal. Thus, the crystal's phase matching angle will have to be adjusted in one direction much larger than the angle of acceptance or angular bandwidth. After the laser is turned off and the crystal cools down to ambient temperature, then the phase matching condition of the crystal will not be satisfied for the same wavelength. Therefore, in order to get the optimum efficiency again, it is very common in high power UV harmonic generation that one has to readjust the angle of the crystal every time when the laser is cycled on and off.

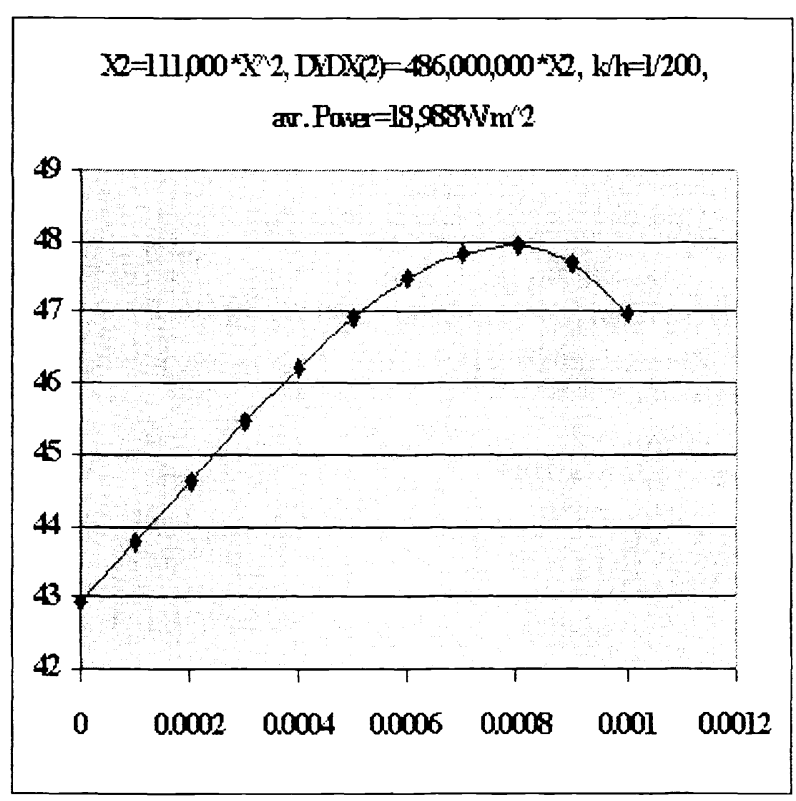

Figure 1a. TPA of 1 UV photon plus a visible photon, $1 \mathrm{~mm}$ crystal length, 3 times the nominal power absorbed (see adjacent manuscript)

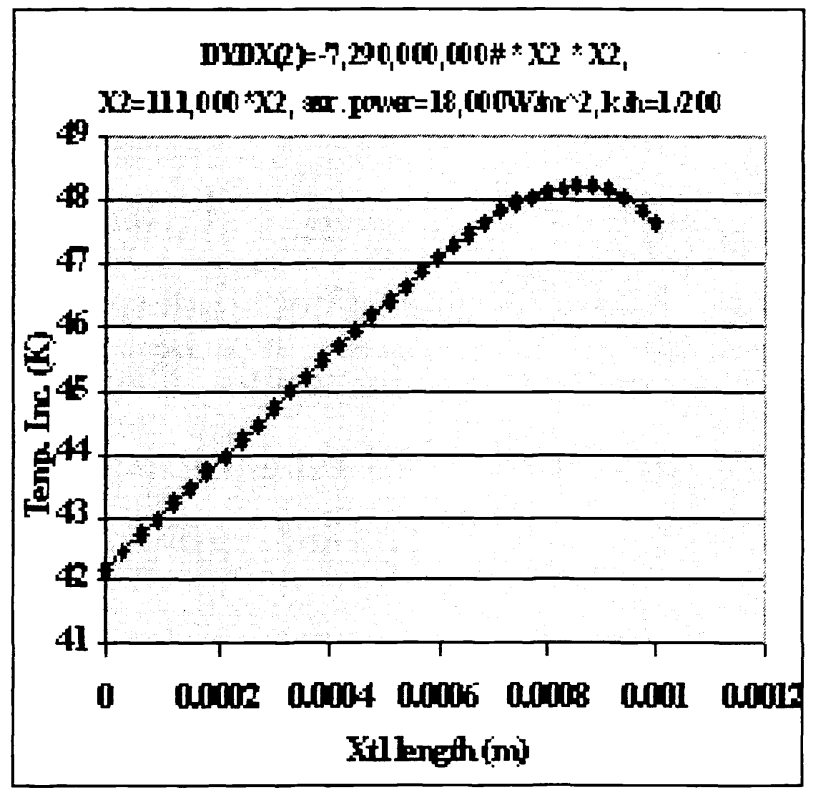

Figure 1b. TPA of 2 UV photon, $1 \mathrm{~mm}$ crystal length, 3 times the nominal power absorbed (see adjacent manuscript)

The temperature gradient is difficult to eliminate by just adjusting the angle of the crystal. Therefore, the temperature gradient will induce a phase mismatch gradient which will degrade the conversion efficiency:

$\delta \mathrm{k}=\delta \mathrm{T} / \Delta \mathrm{T}$, where $\Delta \mathrm{T}$ is the temperature bandwidth of NLO crystal

The compromised conversion efficiency will lower the peak intensity of the deep UV harmonic and reduce the TPA magnitude to a certain degree. And the reduced TPA will alleviate the thermally induced phase mismatch. This kind of interaction has to be included in the 3-wave energy exchange equations for $2^{\text {nd }}$ order nonlinear optical processes.

\section{CALCulation RESUlts}

A computer numerical solution is the practical and straightforward way to simulate such phenomena. We wrote an extra module for the commercial software which does the calculation of the parameters used in nonlinear optical conversions (CASIX NLO Calculator ${ }^{10}$ ) and used it to do some simulation of the instability and efficiency degradation during deep UV harmonic generations. 
Figure 2 demonstrates the SHG efficiency of a BBO crystal calculated by a computer simulation model under different conditions. Plot a shows that under perfect phase matching conditions, $\delta \mathrm{k}=0$, the SHG efficiency increases along the crystal length and approaches unity. Plot $\mathrm{b}$ shows that when $\delta \mathrm{k}$ is small but constant along the crystal length (i.e. there is no thermally induced phase mismatch), the SHG efficiency increases along the crystal length, but cannot reach unit conversion efficiency before back conversion begins. Plot $b$ further reveals that long crystals do not necessarily mean high conversion efficiency, due to beam divergence in the input laser beam. Plot $\mathrm{c}$ presents the case where $\delta \mathrm{k}$ changes because of thermally induced phase mismatch gradient. The efficiency is seen to increase at first, but after reaching a maximum it flattens out as the interaction crystal length increases. This saturation effect has been observed by several research groups ${ }^{5}$. For example, in the generation of the fourth harmonic of the Infinity $\mathrm{Nd}$ :YAG laser, our tests have shown that the efficiency remains nearly constant as the BBO crystal length is increased from 2 to $7 \mathrm{~mm}$. Plot d shows that under heavy thermal loading, when the crystal is angle detuned in the direction that compensates for the thermally induced phase mismatch gradient, the peak efficiency can be increased slightly.

Because BBO and CLBO have a limited temperature bandwidth and their thermal conductivity is not good, the thermally induced gradient can easily generate a phase mismatch that is larger than $\pi n$, where $n$ is the characteristic value under a certain input power. If the thermally induced phase mismatch inside the single crystal is larger than $\pi / n$, then the saturation of conversion efficiency and instability in the harmonic output will be observed. It is therefore essential to use s-BBO (selected$\mathrm{BBO}$ or super-BBO), which has the lowest UV absorption possible cite ${ }^{8}$. Even with more transparent material in the UV, however, thermal loading created by multiphoton absorption still puts a limit on the length of the crystal that can be used.

\section{SHG crystal length v.s. Efficiency}

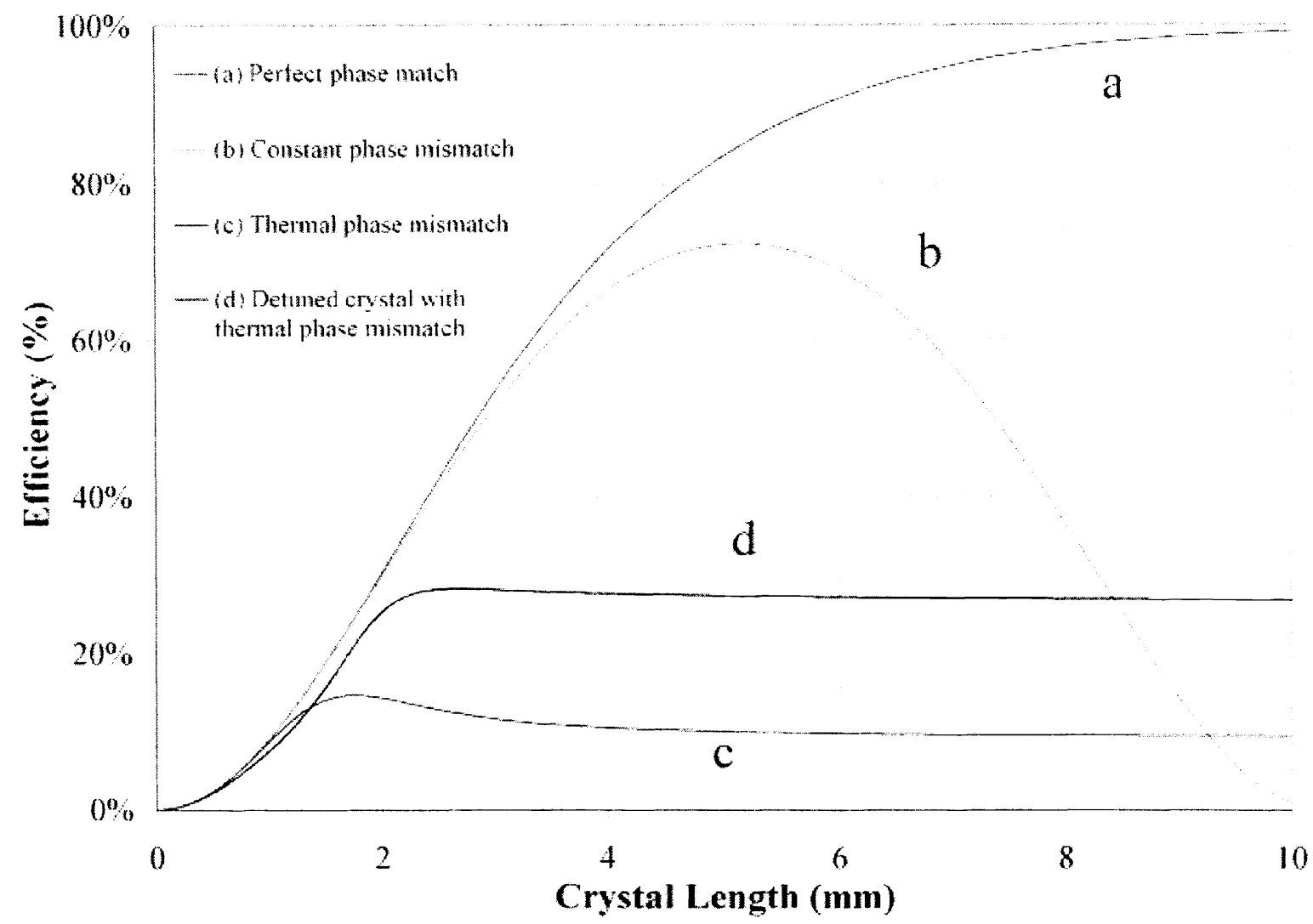

Figure 2. Calculated conversion efficiency inside the NLO crystal under different phase matching conditions. 
For example, newly available Nd:YAG lasers, Infinity, from Coherent (Santa Clara, CA, USA) can power out over 25W at $532 \mathrm{~nm}$ at repetition rate of $100 \mathrm{~Hz}$, 3ns pulse width, $5.5 \mathrm{~mm}$ beam diameter. Under such high loading, one has to reduce the crystal length to $<1 \mathrm{~mm}$ for $\mathrm{BBO}$ and $<3 \mathrm{~mm}$ for CLBO in order to avoid saturation even at low input power. Therefore, the conversion efficiency drops significantly, to about $15 \backslash \%$ for $\mathrm{BBO}$ and $39 \backslash \%$ for CLBO at the full input power, and much lower at smaller input pulse energy. The reason that CLBO crystal has much higher conversion efficiency than BBO crystal under high average power is the direct result of CLBO crystal's relatively larger temperature bandwidth, and possibly much lower two photon absorption $(266 \mathrm{~nm}+532 \mathrm{~nm})$ at $177 \mathrm{~nm}$. We note that the cut off wavelength of CLBO (180nm) is shorter than BBO $(189 \mathrm{~nm})$.

The thermally induced phase mismatch has sign property, the same way as the phase mismatch created by angular detuning, therefore such phase mismatch could be compensated with phase mismatch of the opposite sign. One extreme example of compensation of angular phase mismatch by angular phase mismatch is the periodically poled nonlinear optical material, which are being widely studied recently, where the sign of the crystal principle axis is being altered periodically to cancel the phase mismatch created in the previous period.

The thermally induced phase mismatch gradient discussed above prompts us to separate a single long piece of nonlinear material into several thinner pieces, each angle tuned for optimal phase matching for harmonic generation at the UV loading near full power. The length of each of the thinner crystal is such that the phase mismatch generated by the temperature gradient inside is smaller than the characteristic value $\pi / \mathrm{n}$. Each crystal is angle tuned to compensate for the higher temperature resulting from the increased UV output from the previous crystal and to cancel the phase mismatch created in the previous one. The first crystal is angle tuned for optimal UV generation under ambient or room temperature.

\section{EXPERIMENTAL RESULTS}

We tested our design by using a single $1 \mathrm{~mm}$ piece of $\mathrm{BBO}$, two pieces of $1 \mathrm{~mm} \mathrm{BBO}$ and a single $2 \mathrm{~mm}$ piece of $\mathrm{BBO}$. All of the $\mathrm{BBO}$ crystals were cut for type I phase matching for 4th harmonic generation of $1064 \mathrm{~nm}$, super polished, and coated to reduce the reflection loss at both $532 \mathrm{~nm}$ and $266 \mathrm{~nm}$ to $<2 \%$ from each face. In the two crystal arrangement, care was taken to arrange the direction of the second crystal so that it has the same sign of the nonlinear coefficient $d_{\text {eff }}$ as the first one. The distance between the two crystals was kept under $1 \mathrm{~mm}$ in order to minimize the phase mismatch induced by the dispersion of air.

Figure 3 plots the $532 \mathrm{~nm}$ into $266 \mathrm{~nm}$ conversion efficiencies obtained with a single $1 \mathrm{~mm}$ piece of BBO and with two $1 \mathrm{~mm}$ BBO crystals. At lower input pulse energy $(31 \mathrm{~mJ} /$ pulse at $532 \mathrm{~nm})$, the efficiency of the two crystal setup is roughly 3.8 times that of the single crystal implementation. This demonstrates that the two BBO crystals are working as amplifiers, not independently. The overall efficiency of the two piece setup ranges from $30 \%$ to 2.8 fold over that of the single piece $1 \mathrm{~mm}$ harmonic generator. The reason that the increase in efficiency drops rapidly as input power rises is because the thermally induced phase mismatch becomes sizable, especially in the 2 nd crystal. A shorter crystal should therefore be used for the 2 nd crystal at full power.

Figure 3 also plots the $532 \mathrm{~nm}$ into $266 \mathrm{~nm}$ conversion efficiency obtained with a singe $2 \mathrm{~mm}$ BBO crystal. The efficiency saturates at moderate power input and then decreases quickly as the input power is increased, demonstrating that the thermally induced phase mismatch back converts the $266 \mathrm{~nm}$ harmonic generated inside the crystal. Before saturation, the efficiency of the single $2 \mathrm{~mm}$ crystal is slightly higher than that of the two piece design due to Fresnel reflection losses in the two crystal setup. As the UV power increases, however, thermally induced phase mismatch dominates the conversion efficiency and the two piece harmonic generator outperforms the single $2 \mathrm{~mm}$ crystal. As shown in Figure 3 , at the same 532 $\mathrm{nm}$ input power of $21 \mathrm{~W}$, the two crystal harmonic generator produces over $4.52 \mathrm{~W}$ of $266 \mathrm{~nm}$ light. The single $1 \mathrm{~mm}$ BBO crystal generates $3.20 \mathrm{~W}$, while the $2 \mathrm{~mm}$ crystal produces only $3.05 \mathrm{~W}$. At the maximum $532 \mathrm{~nm}$ power of $27 \mathrm{~W}$, the two crystal harmonic generator produces $5.42 \mathrm{~W}$, while the single $1 \mathrm{~mm}$ output is only $3.70 \mathrm{~W}$. 


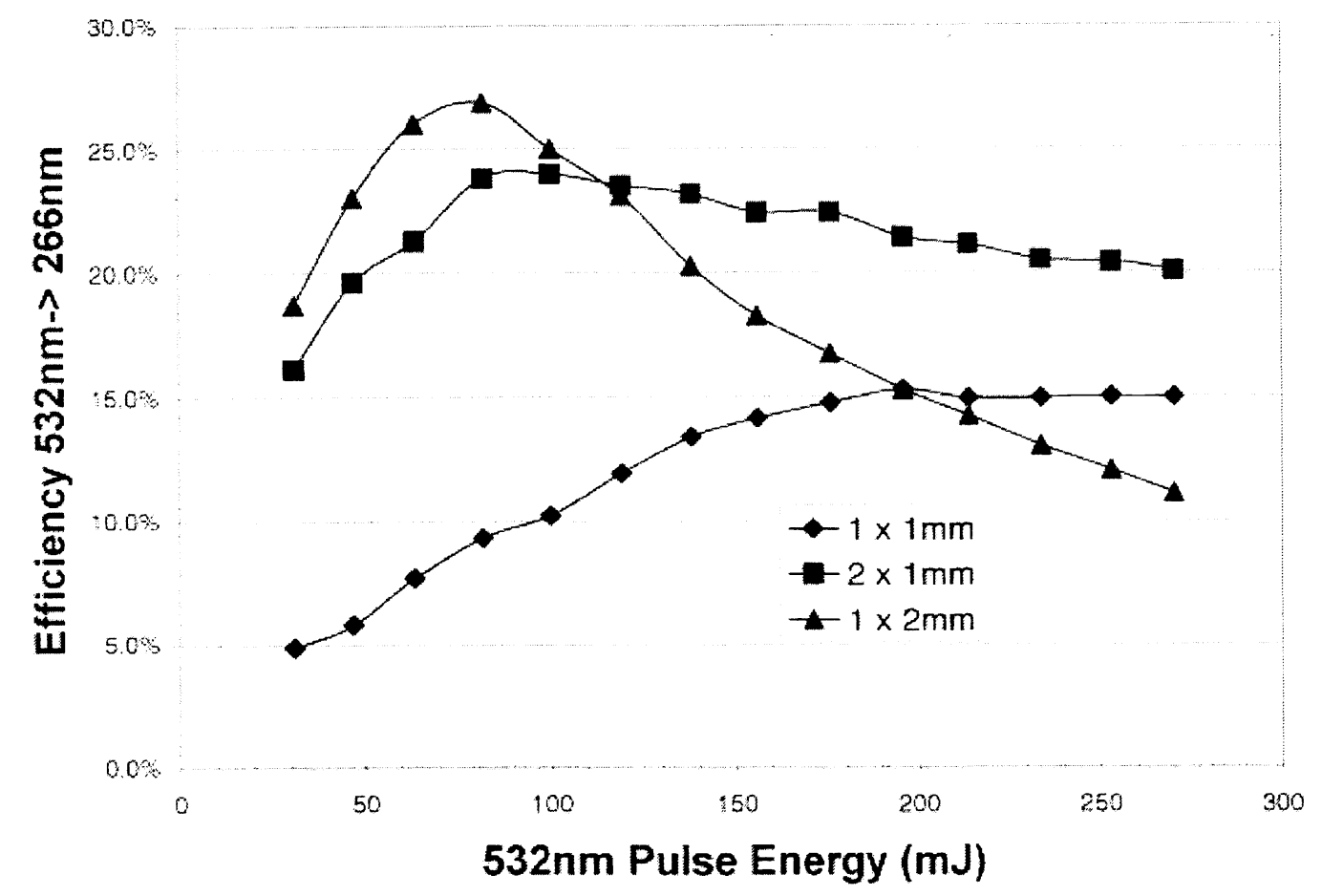

Figure 3 . Measured $4^{\text {th }}$ harmonic $(266 \mathrm{~nm})$ power with 3 different crystal setups.

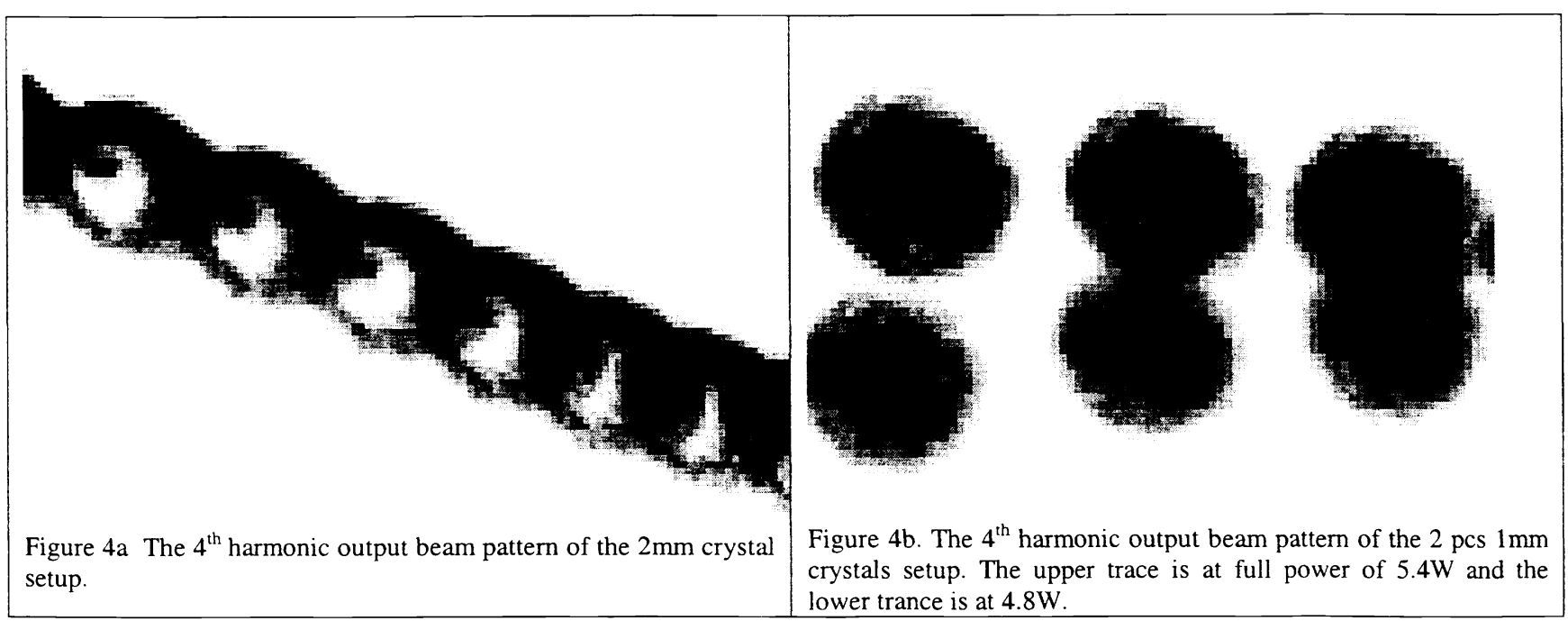


As expected, in order to generate over $3 \mathrm{~W}$ output at $266 \mathrm{~nm}$ with the $2 \mathrm{~mm} \mathrm{BBO}$ crystal, it must be detuned by a large angle from its optimum position at low UV output. The detuned angle is so large that if the laser power is cycled, with sufficient time to allow the $\mathrm{BBO}$ crystal to cool, the UV output power remains less than $0.05 \mathrm{~W}$. Only when the crystal orientation is reoptimized again does the output power return to previous levels, illustrating the unstable nature of the thermally induced phase mismatch. Even at a UV output of $2.5 \mathrm{~W}$, the $2 \mathrm{~mm}$ crystal must be reoptimized after every on/off cycle. With the two crystal harmonic generator, the same UV power (up to $5.42 \mathrm{~W}$ ) can be achieved every time without adjustment. The beam shapes of the $266 \mathrm{~nm}$ pulses at $>2.5 \mathrm{~W}$ output are also quite different for the three different harmonic generators. The single 2 $\mathrm{mm}$ crystal produces a round beam but with very low intensity in the center (see figure 4)., while the $1 \mathrm{~mm}$ and two crystal setups each produce round beams and a relatively uniform intensity distribution.

\section{CONCLUSIONS}

Clearly, there is a substantial improvement in the efficiency, beam profile and repeatability of high power UV harmonic generation in $\mathrm{BBO}$ crystals if multi-crystal designs are used to compensate for the thermally induced phase mismatch. The same phenomenon should be observed with other non-linear optical crystals, such as CLBO and PPLN, and at other harmonic wavelengths. The design presented thus promises a route to increased UV power generation with all solid state sources.

Further work is underway to test crystals with proper lengths and designs with more than two crystals, and a more complete computer simulation model is also being developed. With an optimized suite of crystals, preliminary calculations indicate that each additional crystal should increase the conversion efficiency or the UV power by the amount achievable with a single piece of crystal in the case of the Infinity Nd:YAG laser at full power. Theoretically, with four BBO crystals, over 50\% conversion efficiency from $532 \mathrm{~nm}$ to $266 \mathrm{~nm}$ is feasible at an input power of $25 \mathrm{~W}$ at $532 \mathrm{~nm}$. With even larger YAG lasers, multi-crystal CLBO harmonic generators should be able to generate several tens of Watts of $266 \mathrm{~nm}$ radiation. In practice, the efficiency of such multi-crystal harmonic generators will be limited by the damage threshold of the crystals and especially by the crystal coatings required.

\section{ACKNOWLEDGMENTS}

Funding from NSF MRI program (grant ATM-9724500, sponsored by Atmospheric Chemistry), which provided the pump laser used in these experiments, is gratefully acknowledged.

\section{REFERENCES}

1. Y.K. YAP, K. Deki, N. Kitatochi, Y. Mori and T. Sasaki, Optics Letters, 23, 1016-1018 (1998).

2. Y.K Yap, Y. Mori, S. Haramura, A. Taguchi, T. Sasaki, K.Deki, Y.Ohsako and M. Horiguchi, OSA TOPS Vol.10. 10-13, (1997)

3. U. Stamm, W.Zschocke, T. Schroder, N. Deutsch, D. Basting, OSA TOPS Vol.10, 7-9, (1997)

4. J.K.Tyminski, C.D. Nabors, G.Frangineas, and D.K.Negus, OSA ASSL, Paper MD2, (1995)

5. S.C. Matthews and J.S. Sorce, SPIE, Proceeding. 1220, 137-147 (1990)

6. D. T. Hon and H. Bruesselbach, IEEE J. Quantum Electron, QE-16, 1356--1364, (1980).

7. D. Eimerl, IEEE J. Quantum Electron, QE-23, 575--592 (1987)

8. Data sheet from CASIX, available upon request.

9. R. I. Trickett, M. J. Withford and D. J. Brown, Optics Letters, 23, 189-191, (1998).

10. available at www.u-oplaz.com/NLOCalc.htm 